\title{
AUDIT OF RADIATION DOSE TO PATIENTS DURING CORONARY ANGIOGRAPHY
}

ROSHAN S. LIVINGSTONE, SUNIL CHANDY*, TIMOTHY B. S. PEACE, PAUL V. GEORGE*, BOBBY JOHN ${ }^{*}$, PURENDRA PATI

\section{ABSTRACT}

BACKGROUND: There is widespread concern about radiation doses imparted to patients during cardiology procedures in the medical community. The current study intends to audit and optimize radiation dose to patients undergoing coronary angiography (CA) performed using two dedicated cardiovascular machines. MATERIALS AND METHODS: One hundred and forty nine patients who underwent CA are reported in this study. Dose auditing was done by implementing dose reduction strategies using spectral filters and by evaluating work practices of operators involved in performing CA. SATISTICAL ANALYSIS: A Student's ' $t$ ' test was used to analyze the statistical significance. RESULTS AND CONCLUSION: The radiation dose imparted to patients was measured using dose area product (DAP) meter. The mean DAP values during CA before optimization was $55.86 \mathrm{GY} \mathrm{cm}^{2}$ and after optimization was 27.71 $G y \mathrm{~cm}^{2}$. No ill-effects of radiation were reported for patients who underwent CA. Use of copper filtration may be recommended for procedures performed using cardiovascular machines.

Key words: Dose audit, optimization, radiation dose

\section{INTRODUCTION}

Coronary angiography (CA) constitutes a significant proportion of cardiac investigations performed in cardiac catheterization labs. Considering the fact that catheterization laboratories contribute a large part of manmade radiation exposure to patients compared to diagnostic radiology, radiation dose to patients should be kept as low as possible with acceptable image quality..$^{[1,2]}$

Departments of Radiology and *Cardiology, Christian Medical College, Vellore - 632004 , India

\section{Correspondence}

Assessment of radiation doses to patients undergoing angiography examination is therefore of great importance by virtue of the fact that such procedures can impart large amount of radiation dose to patients. ${ }^{[3]}$

CA involving fluoroscopy and cine runs are invariably performed using dedicated cardiovascular machines. Some of these machines are also equipped with dose area product (DAP) meters for measuring radiation dose imparted to patients. Since exposure parameters varies throughout the procedure and the $\mathrm{x}$-ray beam is moved over different regions of interest, DAP meters are the preferred method of dose assessment. ${ }^{[4]}$
Dose area product is particularly useful for assessing and comparing radiation dose from fluoroscopy procedures as it provides an useful measure of the overall patient exposure than the measurement of surface dose to particular organs. ${ }^{[5]}$

The length of CA depends on complexity of the disease. The complex the anatomy, longer is the duration of fluoroscopy and number of cine runs acquired. ${ }^{[6]}$ Hence, this procedure is likely to impart high radiation doses if adequate radiation safety standards are not undertaken. It is therefore prudent to perform dose auditing on this procedure by implementing radiation safety protocols, ensuring good work practices and comparing risks involved with benefits obtained. Dose auditing during radiological procedures is of great value in optimizing radiation dose to patients without compromising diagnostic yield..$^{[7]}$

\section{MATERIALS AND METHODS}

This study on audit of radiation dose imparted to patients during CA included collection of data over a period of one year. Coronary angiography procedures were performed in two cardiac catheterization labs (P1 and P2) using Philips Integris H3000 and H5000 (Netherlands) machines which were dedicated for cardiovascular procedures. These machines incorporated preset exposure parameters to suit the region of anatomical interest.

There were also several selection modes with specific tabletop dose rates such as low, normal and high for fluoroscopy in both these machines. In the initial stages of the dose auditing, tabletop dose rates were same in both the systems. The dose rates for low

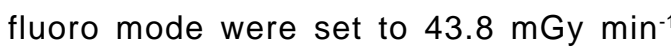
normal to $87.5 \mathrm{mGy} \mathrm{min}^{-1}$ and high to 175 mGy min $^{-1}$. The Philips H5000 machin incorporated spectral filters (added filters with $0.1 \mathrm{~mm}, 0.2 \mathrm{~mm}$ and $0.4 \mathrm{~mm}$ Copper). The Philips $\mathrm{H} 3000$ had a minimum filtration of 1.5 $\mathrm{mm} \mathrm{Al}$ and added filtration similar to Philips H5000. These filters were automatically selected by the machine according to the dose rate settings selected by the operator.

Before the optimization process in the $\mathrm{H} 5000$ machine, company specified protocols for the cardiological procedures were used which did not involve use of heavy tube filtration. The optimization process of reducing radiation doses involved halving the dose rates in the H5000 machine to $21.9 \mathrm{mGy} \mathrm{min}^{-1}, 43.8 \mathrm{mG}$ $\mathrm{min}^{-1}$ and $87.5 \mathrm{mGy} \mathrm{min}^{-1}$ for fluoro selection modes of low, normal and high respectively.

This reduction was achieved by increasing the existing filtration of $0.1 \mathrm{~mm} \mathrm{Cu}$ specified by the manufacturer to $0.4 \mathrm{~mm} \mathrm{Cu}$ and by lowering the tube current and increasing the tube potentials. However, image quality was not adversely affected with the presence of additional copper filters and cardiologists did not find it difficult to perform the procedure The optimization using this dose reduction strategy was not possible with the $\mathrm{H} 3000$ machine due to its tube loading capability and its age. During the CA, operators selected the low fluoro mode, however for a caudal view, normal mode was selected. CA was performed under automatic brightness contro in the tube potential and tube current were adjusted. 
In both the machines, image intensifier formats (IIF) with three field sizes such as $23 \mathrm{~cm}, 17 \mathrm{~cm}$ and $14 \mathrm{~cm}$ were available. A $23 \mathrm{~cm}$ IIF was used for fluoroscopy while tracing the path of the catheter from the region of femoral puncture. However, an IIF of $17 \mathrm{~cm}$ was most frequently used during $\mathrm{CA}$. The nominal focus to image intensifier distance ranged from $90 \mathrm{~cm}$ to $120 \mathrm{~cm}$ during this study and this depended on the views taken. Cine runs were acquired during $C A$ with the number of runs depending on the information needed to be elicited for the study. For cine runs, pulse modes such as 12.5 pulse/sec $\left(\mathrm{ps}^{-1}\right)$ and $25 \mathrm{ps}^{-1}$ were available on the machine. A $25 \mathrm{ps}^{-1}$ mode was invariably selected during the procedure. Throughout each CA, tube current, tube potentials, fluoroscopy time, number of cine runs acquired and the DAP values were recorded. Results were entered into a database for subsequent analysis.

\section{Dosimetry}

Radiation dose imparted to patients who underwent CA was measured using DAP meter (Diamentor PTW; Freiburg Germany) which was part of the machine and was fitted on top of the collimator assembly. The DAP meter measured radiation dose contributed from fluoroscopy and cine runs. During the course of the examination, personnel involved in the data collection continuously monitored DAP values for fluoroscopy and cine runs separately. Periodic calibration of the DAP meter was done by company engineers every six months in collaboration with medical physicists.

\section{Coronary angiography}

Coronary angiography is performed on patients suspected to have ischemic coronary artery stenosis based on symptoms and other non-invasive evaluator such as tread mill stress testing and sestamibi myoperfusion scanning. The patients either had chronic stable angina or acute coronary syndromes ranging from non-ST elevation myocardial infarction or acute ST-elevation myocardial infarction.

All the CA procedures included in this study involved femoral artery puncture under local anesthesia followed by cannulation of the same by the Seldinger technique. Through the $6 \mathrm{~F}$ or $7 \mathrm{~F}$ sheath, longer catheters were negotiated over appropriate guide wires to access the left and right coronary arteries. The catheters commonly used for angiography were Judkins left catheter 3.5 or 4 curve for the left coronary artery and Judkins right catheter 3.5 for the right coronary artery. Left ventricular angiogram was done by the insertion of a non-selective pigtail catheter retrograde from descending aorta-ascending aorta into the left ventricle. Angiographic runs were recorded in views ranging from RAO caudal, RAO cranial, LAO cranial and $L A O$ caudal. Abdominal aortogram in the region of the kidneys were performed for a few patients reported with hypertension. This procedure was done to rule out renal artery stenosis which has been reported in about $50 \%$ of the hypertensive patients who has coronary artery disease..$^{[8]}$ Cine runs were generally used during renal study, however, for a few patients, digital subtraction (DS) was used. Choice of these techniques was at the discretion of personnel performing the procedure.

\section{The CA was categorized into three}

\section{groups namely:}

(i) CA: Patients who underwent $\mathrm{CA}$

(ii) $C A$ + renals: Patients reported with hypertension who underwent $C A$ along with cine runs during renal study

(iii) $C A$ + renals (DS): Patients reported with hypertension who underwent $C A$ along with digital subtraction during renal study

\section{RESULTS}

Out of the one hundred and forty nine patients who underwent CA, 124 were male patients and 25 were female patients. The ages of patients ranged from 33 years to 78 years. The average height and weight of patients was $162.2 \mathrm{~cm}$ and $64.66 \mathrm{~kg}$ respectively. Table 1 shows exposure parameters used during CA performed using the two machines. Before the optimization, the tube potential and tube current used during fluoroscopy were similar in both the machines. After the optimization (P2*), the tube potentials were high with low tube currents during fluoroscopy and during cine runs, the exposure factors remained the same before and after optimization. The number of frames acquired during cine runs ranged from 160 to 2378 and this depended upon the skill of the operator and the information needed to be elicited for the study. The fluoroscopy duration for a CA ranged from $0.4 \mathrm{~min}$ to 34.4 $\min$.

Table 2 shows patient related parameters along with DAP values for catheterization labs $P 1, P 2$ and $P 2 *$. The DAP values from fluoroscopy, cine runs and DS acquisitions are separately shown in Table 2. From table 2 , it is evident that the mean DAP values during CA with renals from DS mode was

\section{Table 1: Exposure parameters during coronary angiography procedure}

\begin{tabular}{|c|c|c|c|c|c|c|c|c|c|c|c|c|c|}
\hline \multirow[t]{2}{*}{ Room } & \multirow[t]{2}{*}{ Examination } & \multicolumn{3}{|c|}{$\begin{array}{l}\text { No. of Mean Fluoro } \\
\text { cases parameters }\end{array}$} & \multicolumn{4}{|c|}{ Mean cine parameters } & \multicolumn{4}{|c|}{ Mean DS parameters } & \multirow{2}{*}{$\begin{array}{l}\text { Fluoro time } \\
\text { in min } \\
\text { Mean }\end{array}$} \\
\hline & & & & $m A$ & $k V$ & $m A$ & $\begin{array}{l}\text { No. of runs } \\
\text { (range) }\end{array}$ & $\begin{array}{l}\text { Frames } \\
\text { (range) }\end{array}$ & $k V$ & $m A s$ & $\begin{array}{c}\text { No. of } \\
\text { exposures }\end{array}$ & $\begin{array}{l}\text { Frames } \\
\text { (range) }\end{array}$ & \\
\hline \multirow[t]{5}{*}{$\mathrm{P} 1$} & CA & 18 & 80 & 22 & 74 & 668 & 8.5 & 1121.06 & - & - & - & - & 5.68 \\
\hline & $\mathrm{CA}+$ renal & 10 & 73 & 21.4 & 72 & 614 & $(4-14)$ & $(404-2151)$ & - & - & - & & $(1.6-13.9)$ \\
\hline & $C A+$ renal & 12 & 77 & 22 & 75 & 658 & 7.8 & 827.1 & 74 & 40 & 1.82 & 19.83 & 4.35 \\
\hline & & & & & & & $(4-13)$ & (294-1631) & & & $(1-7)$ & $(9-86)$ & $(0.7-12.3)$ \\
\hline & & & & & & & $\begin{array}{c}8.5 \\
(6-12)\end{array}$ & $\begin{array}{c}1283 \\
(653-2378)\end{array}$ & & & & & $\begin{array}{c}5.04 \\
(2.3-9.5)\end{array}$ \\
\hline \multirow[t]{6}{*}{ P2 } & & 32 & 82 & 21.3 & 78 & 630 & 8.5 & 1144.06 & - & - & - & & 4.54 \\
\hline & $\mathrm{CA}+$ renal & 7 & 75 & 21 & 73 & 572 & $(4-19)$ & $(411-1949)$ & - & - & - & & $(1.2-29.4)$ \\
\hline & $\mathrm{CA}+$ renal & 17 & 79 & 21.2 & 77 & 585 & & 976.86 & 75 & 46 & 1.09 & 12.9 & 3.77 \\
\hline & (DS) & & & & & & $(6-9)$ & $(729-1233)$ & & & $(1-2)$ & $(8-46)$ & $(1.9-8.6)$ \\
\hline & & & & & & & & 1189.88 & & & & & 4.47 \\
\hline & & & & & & & $(5-14)$ & $(542-2084)$ & & & & & $(1.5-11.2)$ \\
\hline \multirow[t]{4}{*}{$\mathrm{P}^{*}$} & & & & & & 686 & 8.14 & 1170 & - & - & - & - & 3.96 \\
\hline & $\mathrm{CA}$ + renal & 13 & 103 & 6 & 81 & 697 & $(5-13)$ & $(424-2376)$ & - & - & - & & $(0.4-10.2)$ \\
\hline & $\mathrm{CA}$ + renal & 12 & 102 & 6 & 82 & 690 & 10 & 1356 & 75 & 53 & 1 & 11.9 & 6.62 \\
\hline & & & & & & & $\begin{array}{c}(7-14) \\
867\end{array}$ & $\begin{array}{c}(160-2147) \\
1180.42\end{array}$ & & & & $(9-15)$ & $\begin{array}{l}(2.2-16.5) \\
9.47\end{array}$ \\
\hline
\end{tabular}


Table 2: Dose area product values during coronary angiography procedure

\begin{tabular}{|c|c|c|c|c|c|c|c|c|}
\hline Room & Examination & $\begin{array}{l}\text { Mean } \\
\text { Age }\end{array}$ & $\begin{array}{l}\text { Mean } \\
\text { Height }\end{array}$ & $\begin{array}{l}\text { Mean } \\
\text { Weight }\end{array}$ & $\begin{array}{c}\text { Fluoro DAP } \\
\text { in Gy } \mathrm{cm}^{2} \\
\text { Mean } \pm S . E \\
\text { (Range) }\end{array}$ & $\begin{array}{c}\text { Cine DAP } \\
\text { in Gy cm } \\
\text { Mean } \pm S . E \\
\text { (Range) }\end{array}$ & $\begin{array}{c}\text { DSDAP } \\
\text { in Gy cm } \\
\text { Mean } \pm S . E \\
\text { (Range) }\end{array}$ & $\begin{array}{c}\text { Total } D A P \\
\text { in Gy } \mathrm{cm}^{2} \\
\text { Mean } \pm S . E \\
\text { (Range) }\end{array}$ \\
\hline P1 & $\begin{array}{l}\mathrm{CA} \\
\mathrm{CA} \text { + renal } \\
\mathrm{CA}+\text { renal (DS) }\end{array}$ & $\begin{array}{l}54 \\
54 \\
58\end{array}$ & $\begin{array}{l}164 \\
159 \\
165\end{array}$ & $\begin{array}{l}65 \\
57 \\
64\end{array}$ & $\begin{array}{c}15.72 \pm 2.9 \\
(3.4-50.1) \\
7.79 \pm 1.24 \\
(1-16) \\
11.55 \pm 1.37 \\
(6-21)\end{array}$ & $\begin{array}{c}37.04 \pm 3.07 \\
(14.1-59.7) \\
22.67 \pm 2.84 \\
(6.9-33.4) \\
41.37 \pm 5.82 \\
(23.8-91.3)\end{array}$ & $\begin{array}{c}- \\
- \\
- \\
12.08 \pm 3.31 \\
(3.7-38.7)\end{array}$ & $\begin{array}{c}50.56 \pm 5.83 \\
(11.7-96.7) \\
30.46 \pm 3.83 \\
(7.9-43.3) \\
63.24 \pm 7.96 \\
(35.5-127)\end{array}$ \\
\hline P2 & $\begin{array}{l}\mathrm{CA} \\
\mathrm{CA} \text { + renal } \\
\mathrm{CA}+\text { renal (DS) }\end{array}$ & $\begin{array}{l}55 \\
62 \\
55\end{array}$ & $\begin{array}{l}161 \\
160 \\
162\end{array}$ & $\begin{array}{l}66 \\
59 \\
70\end{array}$ & $\begin{array}{c}13.65 \pm 3.03 \\
(2.2-87.8) \\
9.33 \pm 2.18 \\
(4.7-21.8) \\
12.26 \pm 2.06\end{array}$ & $\begin{array}{c}42.2 \pm 4.08 \\
(5.4-103) \\
33.37 \pm 4.79 \\
(22.4-50.7) \\
40.57 \pm 4.93\end{array}$ & $\begin{array}{c}10.26 \pm 1.54 \\
(4.5-26.4)\end{array}$ & $\begin{array}{c}55.86 \pm 5.75 \\
(19.8-148.7) \\
42.7 \pm 7.01 \\
(27.8-72.5) \\
63.1 \pm 7.7\end{array}$ \\
\hline $\mathrm{P} 2^{\star}$ & $\begin{array}{l}\mathrm{CA} \\
\mathrm{CA} \text { + renal } \\
\mathrm{CA}+\text { renal (DS) }\end{array}$ & $\begin{array}{l}57 \\
57 \\
59\end{array}$ & $\begin{array}{l}164 \\
162 \\
163\end{array}$ & $\begin{array}{l}66 \\
69 \\
66\end{array}$ & $\begin{array}{c}(3.8-32.6) \\
5.53 \pm 1.13 \\
(0.6-15.4) \\
9.62 \pm 1.94 \\
(2-25.4) \\
15.23 \pm 3.91 \\
(3.1-42.5)\end{array}$ & $\begin{array}{c}(7-80.1) \\
22.18 \pm 2.02 \\
(11.4-57.2) \\
26.02 \pm 3.5 \\
(12.2-58.3) \\
22.87 \pm 2.02 \\
(11.1-33.4)\end{array}$ & $\begin{array}{c}10.06 \pm 1.16 \\
(5.4-18.1)\end{array}$ & $\begin{array}{c}(16-125.2) \\
27.71 \pm 2.49 \\
(12.6-68.9) \\
35.64 \pm 4.13 \\
(16.2-65.4) \\
46.13 \pm 6.68 \\
(22-90.6)\end{array}$ \\
\hline
\end{tabular}

\section{P2*optimized}

higher than those performed with cine runs. The percentage contribution of radiation dose from cine runs was higher than those from fluoroscopy. The student t-test showed that DAP values from P2 for CA was significantly higher than that for $\mathrm{P}^{*}(P<0.001)$. After the optimization, DAP for cine runs in $P 2^{*}$ were reduced despite similar exposure parameters and number of frames acquired and this was due to the presence of spectral filters. Figure 1 shows the graphical comparison of DAP values before and after optimization for the Philips H5000.

\section{DISCUSSION}

This study was intended to evaluate radiation doses imparted to patients during coronary angiography and to develop methods to optimize the same. Among all the procedures performed in the cardiology department, the number of CA performed was generally high. number of cine runs for CA varied from 4 to 19 [Table 1]. Significant dose reduction is possible if standard protocols are strictly followed by cardiologists performing the procedures; however, it was not feasible to rigorously adhere to standard protocols in all cases due to anatomical variations and complexity of the disease.

The number of frames acquired during cine runs varied from 404 to 2376 . The increase in number of frames was due to the use of $25 \mathrm{ps}^{-1}$ and the duration of each run. As pointed out by Kuon et al, use of a $25 \mathrm{ps}^{-1}$ would also impart low radiation dose to patients if adequate radiation safety standards are maintained. ${ }^{[9]}$ Table 1 shows that during $\mathrm{CA}$, the maximum fluoroscopy time duration was $34.4 \mathrm{~min}$ and the lowest was $0.4 \mathrm{~min}$. The length of CA depended mostly on the pathology of the patient and skill of personnel performing the procedure.

During the optimization process, adequate copper filtration with high tube potentials and low tube currents were selected. It has been observed that with suitable thickness of copper filtration combined with optimized exposure factors, absorbed dose during fluoroscopic procedure can be reduced with little or no loss of image quality. ${ }^{[10]}$ As pointed out by Fenner et al, there is a reduction of $40 \%$ of absorbed dose with only minimal loss of image quality with the use of $0.2 \mathrm{~mm} \mathrm{cu}$ filter. ${ }^{[11]}$ Use of tube potentials of 120 to 125 $\mathrm{kV}$ instead of 90 to $100 \mathrm{kV}$ and corresponding $\mathrm{mAs}$ could reduce entrance surface doses by $50 \%$ and effective dose by $43 \% .^{[12]}$

It is noteworthy in this context that after the optimization, there was a reduction of radiation doses upto $50 \%$ for patients who underwent $\mathrm{CA}$ in cath lab P2* [Table 2]. However in a few cases, radiation doses were high due to the increase in number of cine runs and fluoroscopy time. In light of the above, though there is a dose reduction strategy involved in optimizing doses, it is also possible to impart high radiation doses to patients if appropriate safety standards are not followed. As reported by Kuon et al, it is possible to establish radiation doses as low as 6.2 Gy $\mathrm{cm}^{2}$ with the use of optimal exposure parameters. ${ }^{[13]}$ Radiation doses of the order of 106.3 Gy $\mathrm{cm}^{2}$ has been reported earlier in literature. ${ }^{[14]}$ In the current study, the DAP values recorded in P2 lab ranged from 16 Gy $\mathrm{cm}^{2}$ to $148.7 \mathrm{~Gy} \mathrm{~cm}^{2}$ for CA including those involving renal studies. After the optimization $\left(P 2^{*}\right)$, the DAP values recorded for CA including those involving renal studies ranged from 12.6 Gy $\mathrm{cm}^{2}$ to $90.6 \mathrm{~Gy}_{\mathrm{cm}^{2}}$. In comparison with other studies in literature, the mean DAP values reported by Bakalyar et al, Broadhead et al, Vano et al and Clark et al were 95.2 $\mathrm{Gy} \mathrm{cm}^{2}, 47.7 \mathrm{~Gy} \mathrm{~cm} \mathrm{~cm}^{2}, 66.5 \mathrm{~Gy} \mathrm{~cm}^{2}$ and 20.3 Gy $\mathrm{cm}^{2}$ respectively. ${ }^{[15-18]}$ A strict comparison of literature values is however not possible due to the differences in X-ray systems and the procedure involved.

Major factors contributing to the overall radiation dose would be from the use of high dose rates present in these machines and the length of the fluoroscopy. The cardiovascular machines dedicated for cardiac procedures are invariably imported from developed countries and the default preset exposure settings are based on the patient size of those specified locations. Since the weight and 
body surface area on Indian patients are lower in comparison with patients of other countries, it would be advisable to setup appropriate dose modes for the Indian population. To achieve low dose levels during cardiac procedures, the image intensifier entrance dose rates could be reduced according to the body mass index of the Indian population. However, radiation intensive angulations do not enable substantially better image quality despite increasing image intensifier dose levels. ${ }^{[9]}$ In the current study also, optimization did not result in any significant reduction of image quality. It is noteworthy in this context that thin patients should be examined at low dose levels. Selection of appropriate protocols (optimized and factors meant for pediatric patients) with corresponding dose levels should be encouraged in order to obtain radiation doses as low as reasonably achievable (ALARA) without sacrificing the image quality.

The dose reduction achieved by these methods is likely to translate into low doses to the operators as well. Maintenance and quality assurance checks at regular intervals are mandatory. Improperly calibrated machines can also inadvertently impart large doses to patients without the knowledge of the operator. ${ }^{[19]}$ With a view to maximize the diagnostic benefit and minimize the associated risk from radiation, it is desirable that all patients undergoing angiographic procedures are individually monitored and are regularly followed up. Radiation dose imparted to patients should then be compared with the reference dose levels set by regulatory bodies. These reference dose levels are presently available for developed countries; this can also be adopted in developing countries. In accordance with the ALARA principle, interventionists should therefore vary cine image intensifier dose rates and accept less than optimal image quality in accordance with diagnostic and therapeutic requirements, documented structure, tube angulation and body mass index. ${ }^{[9]}$

\section{CONCLUSION}

Though the radiation dose imparted to patients does not present any alarming situation with regard to ill-effects of radiation, it would be prudent to optimize radiation dose to patients undergoing $C A$. Since there is a frequent change in the various imaging modalities, reference dose levels should be audited on a time to time basis so as to keep the doses as low as reasonably practical. Reduction of radiation dose to patients and consequent minimization of overall radiation dose during $C A$ in the current study was due to use of adequate copper filters with optimal exposure parameters.

\section{ACKNOWLEDGEMENT}

Authors would like to thank all the staff in both the catheterization labs who co-operated with the team for collecting the data.

\section{REFERENCES}

1. Paisley EM, Eatough JP, Mountford PJ, Frain G, Pickerill J. Patient radiation doses during invasive cardiac procedures categorized by clinical code. Br J Radiol 2004;77:1022-6.
2. Broadhead DA, Chapple CL, Faulkner K, Davies $\mathrm{ML}, \mathrm{McC}$ allum $\mathrm{H}$. The impact of cardiology on collective dose in the North of England. Br J Radiol 1997;70:492-7.

3. Hynes DM, Gershater R, Edmonds EW, Rowlands JA, Baranoski D, Turow DG. Radiation dose implications of digital angiographic systems. Am J Roent 1984;143:307-12.

4. Warren-Forward HM, Haddaway MJ, Temperton $\mathrm{DH}, \mathrm{McC}$ all IW. Dose area product readings for fluoroscopic and plain film examinations, including an analysis of the source of variation for barium enema examinations. Br J Radiol 1998;71:961-7.

5. Broadhead DA Chapple CL, Faulkner K. The impact of digital imaging on patient doses during barium studies. Br J Radiol 1995;68:992-6.

6. Kuon E, Glaser C, Dahm JB. Effective techniques for reduction of radiation dosage to patients undergoing invasive cardiac procedures. $\mathrm{Br} \mathrm{J}$ Radiol 2003;76:406-13.

7. Livingstone RS, Justus A, Shyam Kumar NK, Victor RD. Dose audit for radiation safety during intravenous urography procedures using computed radiography imaging system. $\mathrm{J}$ Med Physics 2005;27:304-8.

8. Jaganadha V, Jaishankar S, Raghu K, Sharadha K, Krishna LS, Goutami V, et al. Incidence of renal artery stenosis in coronary artery disease. Indian Heart J 2001;53:61.

9. Kuon E, Dorn C, Schmitt M, Dahm BJ. Radiation dose reduction in invasive cardiology by restriction to adequate instead of optimized picture quality. Health Phys 2003;84:626-31.

10. Tapiovaara MJ, Sandborg M, Dance DR. A search for improved technique factors in paediatric fluoroscopy. Phys Med Biol 1999;44:537-59.

11. Fenner JW, Morrison GD, Kerry J, West N. A practical demonstration of improved technique factors in paediatric fluoroscopy. $\mathrm{Br} \mathrm{J}$ Radiol 2002;75:596-602.

12. Martin CJ, Hunter S. Reduction of patient doses from barium meal and barium enema examinations through changes in equipment factors. $\mathrm{Br} \mathrm{J}$ Radiol 1994;67:1196-205.

13. Kuon E, Schmitt M, Dahm JB. Significant reduction of radiation exposure to staff during cardiac interventions by analysis of radiation leakage and improved lead shielding. Am J Cardiol 2002;89:449.

14. van de Putte S, Verhaegen F, Taeymans $Y$, Thierens $\mathrm{H}$. Correlation of patient skin doses in cardiac interventional radiology with dose-area product. Br J Radiol 2000;73:504-13.

15. Bakalyar DM, Castellani MD, Safian RD. Radiation exposure to patients undergoing diagnostic and interventional cardiac catheterization procedures. Cathet Cardiovasc Diagn 1997;42:121-5.

16. Broadhead S, Chapple CL, Faulkner K, Davies ML, McCallum $H$. The impact of cardiology on the collective effective dose in the North of England. $\mathrm{Br}$ J Radiol 1997;70:492-7.

17. Vano E, Gonzalez L, Fernandez JM, Guibelalde E. Patient dose values in interventional radiology. $\mathrm{Br} \mathrm{J}$ Radiol 1995;68:1215-20.

18. Clark AL, Brennan AG, Robertson LJ, McArthur JD. Factors affecting patient radiation exposure during routine coronary angiography in a tertiary referral centre. Br J Radiol 2000;73:184-9.

19. Livingstone RS, Raghuram L, Korah IP, Raj DV. Evaluation of radiation risk and work practices during cerebral interventions. J Radiol Prot 2003;23:327-36.

Source of Support: Nil, Conflict of Interest: None declared. 